\title{
Modelling and control of a small-scale unmanned helicopter
}

\author{
J F Du ${ }^{1 *}$, K Kondak ${ }^{2}$, Y O Zhang ${ }^{1}$, and T S Lu ${ }^{1}$ \\ ${ }^{1}$ School of Mechanical Engineering, Shanghai Jiao Tong University, Shanghai, People's Republic of China \\ ${ }^{2}$ Faculty of Electrical Engineering and Computer Science, Technische, Universitat Berlin, Berlin, Germany
}

The manuscript was received on 14 January 2008 and was accepted after revision for publication on 31 March 2008.

DOI: 10.1243/09596518JSCE550

\begin{abstract}
The dynamical model of a toy helicopter considered as two rigid bodies is deduced using Kane's equation. Another model is presented that considers the helicopter as a single rigid body. It is shown that the response of the rotational dynamics modelled as two rigid bodies is cosine while that modelled as one rigid body is linear. In addition, a flight controller is presented that is based on dynamic inversion and model predictive control (MPC). In order to decrease the online computational effort associated with a conventional model predictive controller, an explicit MPC algorithm is introduced, which converts the online computations to offline computations to solve the real-time problem. Experimental results show that the controller is able to operate in real-time and can closely track the trajectory without overshoot.
\end{abstract}

Keywords: helicopter, MPC, flight control, Kane's equation

\section{INTRODUCTION}

Small-scale unmanned helicopters, also called toy helicopters, are widely used in many application fields because of their small size and superior flight characteristics, such as vertical take-off, landing, and hovering. They can be used in search and rescue after natural disasters, patrol and surveillance, filming movies, suppression of smuggling, inspection of power lines, large bridges, dams, and so on. However, helicopter models are strongly coupled, multivariate, time delay non-linear systems. The design of a control system for autonomous flight is therefore a challenging task [1, 2].

There is a considerable literature on the modelling of helicopters, but the focus is on large-scale helicopters [3-10]. There are a few papers on toy helicopters. In [5], a non-linear mathematical model for a toy helicopter was deduced in terms of the interaction between the flybar and the main rotor blade and fuselage movement. In [6], a model of a Yamaha-R50 toy helicopter was derived. In [7], a subspace state space method was used to create a model. In [8], a

*Corresponding author: School of Mechanical Engineering, Shanghai Jiao Tong University, Dongchuan Road 800, Shanghai 200240, People's Republic of China.email: djf2717@163.com universal parameter model of roll and pitch channels was developed that considered the main rotor, the flybar, and the fuselage. However, in all of these works, the inertial effects of the main rotor are neglected. In [9] and [10], the authors analysed the inertial effect of the main rotor and found that it makes a significant contribution to the dynamical model. The main rotor should be modelled as a rigid body and not as a mass point. However, Euler angles 1-2-3 not yaw-pitch-roll angles are used in the mathematical model. The main advantage of yaw-pitch-roll angles is that they can be controlled separately. If we correct small errors in the yaw, pitch, and roll individually, we can correct them directly because we have achieved the nominal attitude of the helicopter [11]. This concept is used to derive the mathematical model presented in this paper.

Several controller design methods, such as PID, fuzzy control, and neural network control can be used for flight control [12-14]. However, all these methods have one common drawback: they have first to measure the plant output and compare it with the desired output before the control signals can be generated. Thus, the controller output depends on the actual error and short part of the past state trajectory. Compared with these methods, model-based predictive control (MPC) generates the 
control signal based not only on the actual error and past state trajectory but also on the future behaviour of the system. Also, the control rules (gains) change in every control cycle in order to minimize the control error and satisfy the defined constraints (e.g. limits for controller outputs).

MPC makes an explicit use of the model of the plant to obtain the control signal by minimizing an objective function. MPC consists of three steps. 1. Prediction of the plant output at a future time moment by use of the plant model. 2. Calculation of a control sequence by minimizing an objective function, called receding, which means that at each moment the horizon is shifted forwards. 3. Application of the first control signal of the sequence calculated at each step [15]. This is different from traditional optimization control.

In [16], a non-linear predictive controller was designed for a two-degrees-of-freedom (DOF) toy helicopter using simulation. In [17] a model predictive controller was used to control a two DOF toy helicopter. The controller ran on a dedicated PC running the xPC target real-time kernel to solve the timing problems that plague real-time Windows applications.

However, the conventional quadratic program (QP) algorithm demands significant online computational effort, which limits its applicability to fast plants, such as a six DOF helicopter. The explicit MPC converts the online computations to offline computations which significantly decreases the computation time [18]. In this paper, explicit model predictive control algorithms are introduced to control a six DOF toy helicopter.

The paper is organized as follows. Section 2 discusses multivariate state space models for explicit predictive control with constraints and section 3 deduces the kinematical and dynamical model of the helicopter. Section 4 analyses two models: one that considers the helicopter as one rigid body and one that considers it to be two rigid bodies. Section 5 presents the proposed controller. Experiments are shown in section 6 and conclusions and future work are outlined in section 7 .

\section{EXPLICIT MPC ALGORITHM}

Let the state space equation of a plant be

$$
\begin{aligned}
& \boldsymbol{x}(k+1)=\mathbf{A} \boldsymbol{x}(k)+\mathbf{B} \boldsymbol{u}(k) \\
& \boldsymbol{y}(k)=\mathbf{C} \boldsymbol{x}(k)+\mathbf{D} \boldsymbol{u}(k)
\end{aligned}
$$

while fulfilling the constraints:

$$
\begin{aligned}
& \boldsymbol{y}_{\text {min }} \leqslant \boldsymbol{y}(k) \leqslant \boldsymbol{y}_{\text {max }} \\
& \boldsymbol{u}_{\min } \leqslant \boldsymbol{u}(k) \leqslant \boldsymbol{u}_{\max }
\end{aligned}
$$

where, $\boldsymbol{x}(k)$ is the $n$-dimensional state vector of the plant, $\boldsymbol{u}(k)$ is the $m$-dimensional vector of manipulated variables, and $\boldsymbol{y}(k)$ is the $p$-dimension vector of the plant output.

The MPC algorithm minimizes an objective function (2) to obtain the control law

$$
\min \sum_{k=0}^{N-1} \begin{aligned}
& {[\boldsymbol{y}(t+k \mid t)-\boldsymbol{r}(t)]^{\prime} \mathbf{S}[\boldsymbol{y}(t+k \mid t)-\boldsymbol{r}(t)]} \\
& +\Delta \boldsymbol{u}^{\prime}(t+k) \mathbf{T} \Delta \boldsymbol{u}(t+k)
\end{aligned}
$$

subject to

$$
\begin{aligned}
& \boldsymbol{y}_{\min } \leqslant \boldsymbol{y}_{t+k \mid t} \leqslant \boldsymbol{y}_{\max }, \quad k=1, \cdots, N_{c y} \\
& \boldsymbol{u}_{\min } \leqslant \boldsymbol{u}_{t+k} \leqslant \boldsymbol{u}_{\max }, \quad k=0, \cdots, N_{c u} \\
& \Delta \boldsymbol{u}_{\min } \leqslant \Delta \boldsymbol{u}(t+k) \leqslant \Delta \boldsymbol{u}_{\max }, \quad k=0, \cdots N_{c u} \\
& \boldsymbol{u}(t+k)=0, \quad k \geqslant N_{u} \\
& \boldsymbol{x}_{t \mid t}=\boldsymbol{x}(t) \\
& \boldsymbol{x}(t+k+1 \mid t)=\mathbf{A} x(t+k \mid t)+\mathbf{B}[\boldsymbol{u}(t+k-1)+\Delta \boldsymbol{u}(t+k)], \quad k \geqslant 0 \\
& \boldsymbol{y}(t+k \mid t)=\mathbf{C} \boldsymbol{x}(t+k \mid t)+\mathbf{D}[\boldsymbol{u}(t+k-1)+\Delta \boldsymbol{u}(t+k)] \\
& \quad k \geqslant 0
\end{aligned}
$$

where $\Delta \boldsymbol{u}(t)=\boldsymbol{u}(t)-\boldsymbol{u}(t-1)$, $\mathbf{S}$ is the error weight matrix, $\mathbf{T}$ is the control weight matrix, $\boldsymbol{r}$ is the reference input, $N, N_{c y}, N_{c u}, N_{u}$ are the predictive, output constraint, input constraint, control horizon, respectively.

By substituting $\boldsymbol{x}(t+k \mid t)=\mathbf{A}^{k} \boldsymbol{x}(t)+\sum_{j=0}^{k-1} \mathbf{A}^{j} \mathbf{B} \boldsymbol{u}(t+$ $k-1-j)$, equation (2) can be rewritten as

$$
\min \frac{1}{2} \boldsymbol{z}^{\prime} \mathbf{Q} \boldsymbol{z}+\boldsymbol{\theta}^{\prime} \mathbf{C}^{\prime} \boldsymbol{z}
$$

subject to

$$
\mathbf{G} z \leqslant \mathbf{W}+\mathbf{S} \boldsymbol{\theta}
$$

where $z=\left[u^{\prime}(0), \ldots, u^{\prime}(m-1)\right]^{\prime}$ is the vector to be optimized and $\boldsymbol{\theta}=[\boldsymbol{x}(t), \boldsymbol{u}(t-1), r(t)]^{\prime}$ is the vector of parameters, and matrix $\mathbf{Q}, \mathbf{C}, \mathbf{G}, \mathbf{W}, \mathbf{S}$ are easily obtained from equation (2).

For this multi-parametric QP (mp_QP) problem, we need find an initial vector $\boldsymbol{\theta}_{\boldsymbol{o}}$ inside the 
polyhedral set $\Theta=\{\boldsymbol{\theta}: \mathbf{T} \boldsymbol{\theta} \leqslant \mathbf{Z}\}$ of parameters over which we want to solve the problem. This can be solved by the linear program

$$
\max _{\theta, z} \varepsilon
$$

subject to

$$
\begin{aligned}
& \mathbf{T}^{i} \boldsymbol{\theta}+\varepsilon\left\|\mathbf{T}^{i}\right\| \leqslant \mathbf{Z}^{i} \\
& \mathbf{G} \boldsymbol{z}-\mathbf{S} \boldsymbol{\theta} \leqslant \mathbf{W}
\end{aligned}
$$

Then the optimization solution $z_{\boldsymbol{o}}$ can be solved by the QP problem (3) and a set of active constraints $\tilde{\mathbf{G}} z_{\boldsymbol{o}}=$ $\tilde{\mathbf{W}}+\tilde{\mathbf{S}} \boldsymbol{\theta}_{0}$ out of the constraints (3) can be determined.

The QP problem (3) can be solved by applying the Karush-Kuhn-Tuker conditions

$$
\begin{gathered}
\mathbf{Q} z+\mathbf{C} \boldsymbol{\theta}+\mathbf{G}^{\prime} \lambda=0, \lambda \in R^{q} \\
\lambda_{i}\left(\mathbf{G}^{i} \boldsymbol{z}-\mathbf{W}^{i}-\mathbf{S}^{i} \boldsymbol{\theta}\right)=0, i=1, \cdots, q \\
\lambda \geqslant 0 \\
\mathbf{G} \boldsymbol{z}-\mathbf{W}-\mathbf{S} \boldsymbol{\theta} \leqslant 0
\end{gathered}
$$

where, the superscript $i$ denotes the $i$ th row. $\mathbf{Q}$ is a full rank matrix, so we can solve equation (5a) for $z$

$$
z=-\mathbf{Q}^{-1}\left(\mathbf{C} \boldsymbol{\theta}+\mathbf{G}^{\prime} \lambda\right)
$$

Let $\breve{\lambda}$ and $\tilde{\lambda}$ denote the Lagrange multipliers corresponding to inactive constraints and active constraints, respectively. For inactive constraints, $\breve{\lambda}=0$. For active constraints, $-\tilde{\mathbf{G}} \mathbf{Q}^{-1}\left(\mathbf{C} \boldsymbol{\theta}+\tilde{\mathbf{G}}^{\prime} \tilde{\boldsymbol{\lambda}}\right)-\tilde{\mathbf{W}}-\tilde{\mathbf{S}} \boldsymbol{\theta}=0$, and therefore

$$
\tilde{\boldsymbol{\lambda}}=-\left(\tilde{\mathbf{G}} \mathbf{Q}^{-1} \tilde{\mathbf{G}}^{\prime}\right)^{-1}\left[(\tilde{\mathbf{W}}+\tilde{\mathbf{S}} \boldsymbol{\theta})+\tilde{\mathbf{G}} \mathbf{Q}^{-1} \mathbf{C} \boldsymbol{\theta}\right]
$$

where, $\left(\tilde{\mathbf{G}} \mathbf{Q}^{-1} \tilde{\mathbf{G}}^{\prime}\right)^{-1}$ exists because the rows of $\tilde{\mathbf{G}}$ are linearly independent.

By substituting $\tilde{\lambda}$ from equation (7) into equation

(6) it is possible to obtain

$$
\begin{aligned}
& \boldsymbol{z}= \\
& -\mathbf{Q}^{-1}\left\{\mathbf{C} \boldsymbol{\theta}-\tilde{\mathbf{G}}^{\prime}\left(\tilde{\mathbf{G}} \mathbf{Q}^{-1} \tilde{\mathbf{G}}^{\prime}\right)^{-1}\left[(\tilde{\mathbf{W}}+\tilde{\mathbf{S}} \boldsymbol{\theta})+\tilde{\mathbf{G}} \mathbf{Q}^{-1} \mathbf{C} \boldsymbol{\theta}\right]\right\}
\end{aligned}
$$

From equations (7) and (8), we can see that both $\tilde{\lambda}$ and $z$ are affine functions of $\boldsymbol{\theta}$. However, they characterize the solution only locally in the neighbourhood of a specific $\boldsymbol{\theta}_{0}$, since optimization active sets are not produced when this characterization remains valid. This region can be characterized as follows.

First, the region must satisfy the constraints in equation (3)

$$
\begin{aligned}
& -\mathbf{G Q}^{-1}\left\{\mathbf{C} \boldsymbol{\theta}-\tilde{\mathbf{G}}^{\prime}\left(\tilde{\mathbf{G}} \mathbf{Q}^{-1} \tilde{\mathbf{G}}^{\prime}\right)^{-1}\left[(\tilde{\mathbf{W}}+\tilde{\mathbf{S}} \boldsymbol{\theta})+\tilde{\mathbf{G}} \mathbf{Q}^{-1} \mathbf{C} \boldsymbol{\theta}\right]\right\} \\
& \leqslant \boldsymbol{W}+\boldsymbol{S} \boldsymbol{x}
\end{aligned}
$$

Second, the Lagrange multipliers must be nonnegative

$$
-\left(\tilde{\mathbf{G}} \mathbf{Q}^{-1} \tilde{\mathbf{G}}^{\prime}\right)^{-1}\left[(\tilde{\mathbf{W}}+\tilde{\mathbf{S}} \boldsymbol{\theta})+\tilde{\mathbf{G}} \mathbf{Q}^{-1} \mathbf{C} \boldsymbol{\theta}\right] \geqslant 0
$$

Equations (9) and (10) construct a polyhedron $C R_{0}$ which is the largest set such that the combination of active constraints at the minimum remains unchanged. Thus, the solution $z$ is obtained given $\theta_{0}$ in the region $C R_{0}$. As for the rest of $C R_{0}$, it can be partitioned into several subspaces through the following algorithm.

Let $Y \subseteq R^{n}$ be a polyhedron, and $C R_{0}\{\boldsymbol{x} \in \boldsymbol{Y}: \mathbf{A x} \leqslant \boldsymbol{b}\}$ a polyhedral subset of $Y, C R_{0} \neq \emptyset$. Also let

$$
R_{i}=\left\{\boldsymbol{x} \in \boldsymbol{Y}: \begin{array}{c}
\mathbf{A}^{i} \boldsymbol{x}>\mathbf{b}^{i} \\
\mathbf{A}^{j} \boldsymbol{x} \leqslant \mathbf{b}^{j}, \forall j<i
\end{array}\right\}, i=1, \cdots m
$$

where, $m=\operatorname{dim}(\mathbf{b})$, and let $C R^{\text {rest }} \cup_{i=1}^{m} R_{i}$. Then

1. $C R^{\text {rest }} \cup C R_{0}=Y$

2. $C R_{0} \cup R_{i}=\varnothing, R_{i} \cup R_{j}=\varnothing, \forall i \neq j$ i.e. $\left\{C R_{0}, R_{1}, \ldots\right.$, $\left.R_{m}\right\}$ is a partition of $Y$.

For each subspace, the procedure of finding the solution and the optimization active sets is iterated. Finally, every solution is found in every subspace.

More details of the algorithm can be found in [18].

\section{MODELLING OF THE HELICOPTER}

The main differences between toy helicopters and full-size helicopters include the following [10]:

(a) a much higher ratio of the main rotor mass to the fuselage mass;

(b) the rotation speed of the main rotor of toy helicopters is higher than that of most full-sized helicopters; 
(c) toy helicopters have very stiff main rotors without flapping hinges.

The initial effects of the main rotor make a significant contribution to the rotational dynamics and can not be neglected. Not only the fuselage but also the main rotor should be modelled as rigid body.

The simplified model of a toy helicopter is shown in Fig. 1, where the fuselage is labelled with an F, the main rotor with MR, the tail rotor with $\mathrm{TR}$, the mass centre of the fuselage with Fo, the mass centre of the main rotor with MRo, the mass centre of the tail rotor with TRo and the variables are as listed in the Appendix.

The kinematical equations of translation and rotation can be deduced as follows.

The kinematical equations of translation are

$$
\begin{aligned}
& \dot{p}_{x}=u \\
& \dot{p}_{y}=v \\
& \dot{p}_{z}=w
\end{aligned}
$$

The kinematical equations of rotation

$$
\begin{aligned}
& \dot{\phi}=p+\tan (\theta)(\sin (\phi) q+\cos (\phi) r) \\
& \dot{\theta}=\cos (\phi) q-\sin (\phi) r \\
& \dot{\psi}=(\sin (\phi) q+\cos (\phi) r) / \cos (\theta)
\end{aligned}
$$

As for the dynamical equations, the helicopter is regarded as two rigid bodies, the fuselage and the main rotor. Kane's method is chosen for modelling because of its low computational complexity. In order to simplify the dynamics, the following assumptions will be considered.

1. The axis $f_{3}$ is in-line with the main rotor axis and the mass centre of the fuselage is located on $f_{3}$.

2. $r$ is constant.

3. $\omega_{\mathrm{MR}}$ is constant and the moment of inertia of the main rotor $I_{11}^{\mathrm{MR}}$ is equal to $I_{22}^{\mathrm{MR}}$.

4. The products of the inertias of different axes of the fuselage and main rotor are zero.

5. The lifting force and drag force of the horizontal tail, the lateral force, and the drag force of the vertical tail and the aerodynamic force of the fuselage are neglected.

6. The effect of inertia of the tail rotor is neglected.

The first assumption can be achieved by appropriate placement of the equipment on the fuselage and the second assumption can be achieved by

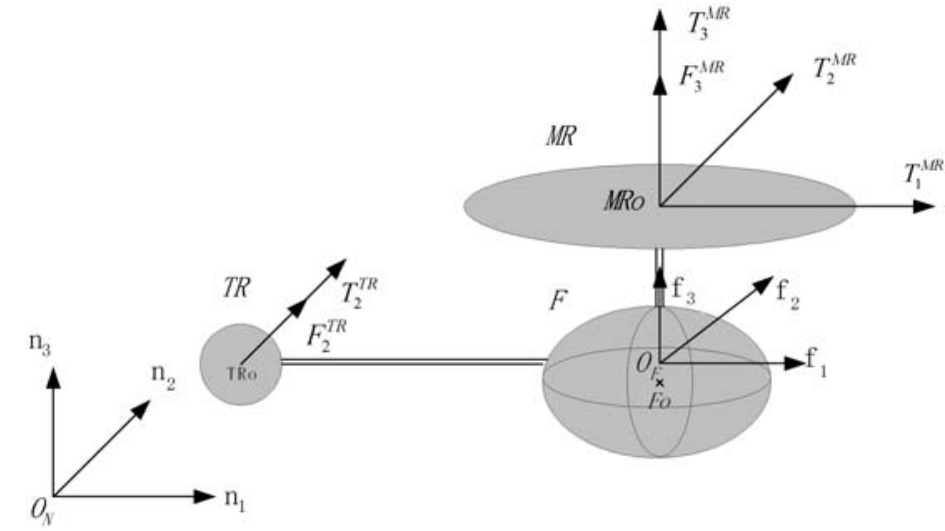

Fig. 1 Simplified model of the toy helicopter

using a separate control loop for $r$. The angular velocity of the main rotor is constant which can also be implemented by using a separated control loop for $\omega_{\mathrm{MR}}$. Thus, the assumptions that $\omega_{\mathrm{MR}}$ is constant are reasonable. As the body of the helicopter is nearly symmetrical we can suggest that the moment of inertia of the main rotor $I_{11}^{\mathrm{MR}}$ is equal to $I_{22}^{\mathrm{MR}}$ and the products of the inertias of different axes of the fuselage and main rotor are zero. Assumption 5 is valid if the helicopter moves at a low speed. Actually in many applications, such as the inspection of power lines, the speed of the helicopter is very slow. Thus, this assumption is reasonable. The effect of the inertia of the tail rotor is very small which has been validated in [10], so it is neglected during the modelling.

The positions of the helicopter $p_{x}, p_{y}, p_{z}$ and the pitch-roll-yaw angles $\phi, \theta, \psi$ completely describe the configuration of the system and are defined as generalized coordinates. The translational speeds $u$, $v, w$ and the rotational speeds $p, q, r$ describe the motion of the system and are defined as generalized speeds. In order to obtain Kane's dynamical equations, the velocities of the mass centres $\mathrm{TR}_{\mathrm{O}}, \mathrm{F}_{\mathrm{O}}$, $\mathrm{MR}_{\mathrm{O}}$ and the angular velocities of the fuselage and main rotor are calculated firstly, then the partial velocities.

The partial velocities of the mass centre of the fuselage, Fo can be obtained as

$$
\begin{aligned}
& \boldsymbol{v}_{1}^{\mathrm{Fo}}=\frac{\partial \boldsymbol{v}^{\mathrm{Fo}}}{\partial u}=\boldsymbol{n}_{1}, \quad \boldsymbol{v}_{2}^{\mathrm{Fo}}=\frac{\partial \boldsymbol{v}^{\mathrm{Fo}}}{\partial v}=\boldsymbol{n}_{2}, \quad \boldsymbol{v}_{3}^{\mathrm{Fo}}=\frac{\partial \boldsymbol{v}^{\mathrm{Fo}}}{\partial w}=\boldsymbol{n}_{3}, \\
& \boldsymbol{v}_{4}^{\mathrm{Fo}}=\frac{\partial \boldsymbol{v}^{\mathrm{Fo}}}{\partial p}=-\left(L_{\mathrm{F}}-L_{\mathrm{MR}}\right) \frac{m_{\mathrm{MR}}}{M} \boldsymbol{f}_{2} \\
& \boldsymbol{v}_{5}^{\mathrm{Fo}}=\frac{\partial \boldsymbol{v}^{\mathrm{Fo}}}{\partial \boldsymbol{q}}=\left(L_{\mathrm{F}}-L_{\mathrm{MR}}\right) \frac{m_{\mathrm{MR}}}{M} \boldsymbol{f}_{1}, \quad \boldsymbol{v}_{6}^{\mathrm{Fo}}=\frac{\partial \boldsymbol{v}^{\mathrm{Fo}}}{\partial r}=0
\end{aligned}
$$

The partial velocities of the mass centre of the tail 
rotor TRo can be obtained as

$$
\begin{aligned}
& \boldsymbol{v}_{1}^{\mathrm{TRo}}=\frac{\partial \boldsymbol{v}^{\mathrm{TRo}}}{\partial u}=\boldsymbol{n}_{1}, \quad \boldsymbol{v}_{2}^{\mathrm{TRo}}=\frac{\partial \boldsymbol{v}^{\mathrm{TRo}}}{\partial v}=\boldsymbol{n}_{2}, \\
& \boldsymbol{v}_{3}^{\mathrm{TRo}}=\frac{\partial \boldsymbol{v}^{\mathrm{TRo}}}{\partial w}=\boldsymbol{n}_{3}, \\
& \boldsymbol{v}_{4}^{\mathrm{TRo}}=\frac{\partial \boldsymbol{v}^{\mathrm{TRo}}}{\partial p}=\frac{\left(L_{\mathrm{F}} m_{\mathrm{F}}+L_{\mathrm{MR}} m_{\mathrm{MR}}\right)}{M} \boldsymbol{f}_{2} \\
& \boldsymbol{v}_{5}^{\mathrm{TRo}}=\frac{\partial \boldsymbol{v}^{\mathrm{TRo}}}{\partial \boldsymbol{q}}=-\frac{\left(L_{\mathrm{F}} m_{\mathrm{F}}+L_{\mathrm{MR}} m_{\mathrm{MR}}\right)}{M} \boldsymbol{f}_{1}+L_{\mathrm{T}} \boldsymbol{f}_{3}, \\
& \boldsymbol{v}_{6}^{\mathrm{TRo}}=\frac{\partial \boldsymbol{v}^{\mathrm{TRo}}}{\partial \boldsymbol{r}}=-L_{\mathrm{T}} \boldsymbol{f}_{2}
\end{aligned}
$$

The partial velocities of the mass centre of the main rotor MRo can be obtained as

$$
\begin{aligned}
& \boldsymbol{v}_{1}^{\mathrm{MRo}}=\frac{\partial \boldsymbol{v}^{\mathrm{MRo}}}{\partial u}=\boldsymbol{n}_{1}, \quad \boldsymbol{v}_{2}^{\mathrm{MRo}}=\frac{\partial \boldsymbol{v}^{\mathrm{MRo}}}{\partial \boldsymbol{v}}=\boldsymbol{n}_{2}, \\
& \boldsymbol{v}_{3}^{\mathrm{MRo}}=\frac{\partial \boldsymbol{v}^{\mathrm{MRo}}}{\partial w}=\boldsymbol{n}_{3}, \\
& \boldsymbol{v}_{4}^{\mathrm{MRo}}=\frac{\partial \boldsymbol{v}^{\mathrm{MRo}}}{\partial p}=-\left(L_{\mathrm{MR}}-L_{\mathrm{F}}\right) \frac{m_{\mathrm{F}}}{M} \boldsymbol{f}_{2} \\
& \boldsymbol{v}_{5}^{\mathrm{MRo}}=\frac{\partial \boldsymbol{v}^{\mathrm{MRo}}}{\partial \boldsymbol{q}}=\left(L_{\mathrm{MR}}-L_{\mathrm{F}}\right) \frac{m_{\mathrm{F}}}{M} \boldsymbol{f}_{1}, \quad \boldsymbol{v}_{6}^{\mathrm{MRo}}=\frac{\partial \boldsymbol{v}^{\mathrm{MRo}}}{\partial r}=0
\end{aligned}
$$

The partial velocities of the fuselage and the main rotor (they have the same partial velocities)

$$
\begin{aligned}
& \omega_{1}^{\mathrm{F}}\left(\omega_{1}^{\mathrm{MR}}\right)=\frac{\partial \omega^{\mathrm{F}}}{\partial u}=0, \quad \omega_{2}^{\mathrm{F}}\left(\omega_{2}^{\mathrm{MR}}\right)=\frac{\partial \omega^{\mathrm{F}}}{\partial \nu}=0, \\
& \omega_{3}^{\mathrm{F}}\left(\omega_{3}^{\mathrm{MR}}\right)=\frac{\partial \omega^{\mathrm{F}}}{\partial \omega}=0 \\
& \omega_{4}^{\mathrm{F}}=\left(\omega_{4}^{\mathrm{MR}}\right)=\frac{\partial \omega^{\mathrm{F}}}{\partial p}=\boldsymbol{f}_{1}, \quad \omega_{5}^{\mathrm{F}}\left(\omega_{5}^{\mathrm{MR}}\right)=\frac{\partial \omega^{\mathrm{F}}}{\partial q}=\boldsymbol{f}_{2}, \\
& \omega_{6}^{\mathrm{F}}\left(\omega_{6}^{\mathrm{MR}}\right)=\frac{\partial \omega^{\mathrm{F}}}{\partial r}=\boldsymbol{f}_{3}
\end{aligned}
$$

The forces and the torques acting on the helicopter are $F_{2}^{\mathrm{TR}}, F_{3}^{\mathrm{MR}}, T_{2}^{\mathrm{TR}}, T_{1}^{\mathrm{MR}}, T_{2}^{\mathrm{MR}}, T_{3}^{\mathrm{MR}}$, and the generalized active forces $F_{r}(r=1,2, \ldots, 6)$ can be calculated by the following equation

$$
\begin{aligned}
F_{r} & =F_{2}^{\mathrm{TR}} \boldsymbol{f}_{2} \cdot v_{r}^{\mathrm{TRo}}+F_{3}^{\mathrm{MR}} \boldsymbol{f}_{3} \cdot v_{r}^{\mathrm{MRo}}+m_{\mathrm{F}} \boldsymbol{g} \boldsymbol{n}_{3} \cdot \boldsymbol{v}_{r}^{\mathrm{Fo}} \\
& +m_{\mathrm{MR}} g \boldsymbol{n}_{3} \cdot v_{r}^{\mathrm{MRo}}+\left(T_{1}^{\mathrm{MR}} \boldsymbol{f}_{1}+T_{2}^{\mathrm{MR}} \boldsymbol{f}_{2}\right. \\
& \left.+T_{3}^{\mathrm{MR}} \boldsymbol{f}_{3}\right) \cdot \boldsymbol{\omega}_{r}^{\mathrm{MR}}+T_{2}^{\mathrm{TR}} \boldsymbol{f}_{2} \cdot \boldsymbol{\omega}_{r}^{F}
\end{aligned}
$$

$$
\begin{aligned}
F_{1}= & F_{3}^{\mathrm{MR}}(\sin (\phi) \sin (\psi)+\sin (\theta) \cos (\phi) \cos (\psi)) \\
& -F_{2}^{\mathrm{TR}}(\sin (\psi) \cos (\phi)-\sin (\phi) \sin (\theta) \cos (\psi)) \\
F_{2}= & F_{2}^{\mathrm{TR}}(\cos (\phi) \cos (\psi)+\sin (\phi) \sin (\theta) \sin (\psi)) \\
& -F_{3}^{\mathrm{MR}}(\sin (\phi) \cos (\psi)-\sin (\theta) \sin (\psi) \cos (\phi)) \\
F_{3}= & F_{2}^{\mathrm{TR}} \sin (\phi) \cos (\theta)+F_{3}^{\mathrm{MR}} \cos (\phi) \cos (\theta)-M g \\
F_{4}= & T_{1}^{\mathrm{MR}}+\frac{L_{\mathrm{MR}} m_{\mathrm{MR}}+L_{\mathrm{F}} m_{\mathrm{F}}}{M} F_{2}^{\mathrm{TR}} \\
F_{5}= & T_{2}^{\mathrm{MR}}+T_{2}^{\mathrm{TR}} \\
F_{6}= & T_{3}^{\mathrm{MR}}-L_{T} F_{2}^{\mathrm{TR}}
\end{aligned}
$$

The generalized inertial forces $F_{r}^{*}(r=1,2, \ldots, 6)$ can be calculated as

$$
F_{r}^{*}=-\left\{\begin{array}{l}
m_{\mathrm{F}} a_{\mathrm{F}}^{\mathrm{Fo}} \cdot v_{r}^{\mathrm{Fo}}+\left[I_{\mathrm{F}} \boldsymbol{\alpha}_{\mathrm{F}}+\omega_{\mathrm{F}} \times\left(I_{\mathrm{F}} \omega_{\mathrm{F}}\right)\right] \cdot \omega_{r}^{\mathrm{F}} \\
+m_{\mathrm{MR}} a_{\mathrm{MR}}^{\mathrm{MRo}} \cdot v_{r}^{\mathrm{MRo}}+\left[\begin{array}{c}
I_{\mathrm{MR}} \boldsymbol{\alpha}_{\mathrm{MR}}+\omega_{\mathrm{MR}} \\
\times\left(I_{\mathrm{MR}} \omega_{\mathrm{MR}}\right)
\end{array}\right] \cdot \omega_{r}^{\mathrm{MR}}
\end{array}\right\}
$$

Thus

$$
\begin{aligned}
F_{1}^{*}= & -M \dot{u}, \quad F_{2}^{*}=-M \dot{v}, \quad F_{3}^{*}=-M \dot{w} \\
F_{4}^{*}= & -\left(I_{11}^{\mathrm{MR}}+I_{11}^{\mathrm{F}}+\frac{m_{\mathrm{F}} m_{\mathrm{MR}}}{M}\left(L_{\mathrm{F}}-L_{\mathrm{MR}}\right)^{2}\right) \dot{p} \\
& +\left(I_{22}^{\mathrm{F}}-I_{33}^{\mathrm{F}}-I_{11}^{\mathrm{MR}}+\frac{m_{\mathrm{F}} m_{\mathrm{MR}}}{M}\left(L_{\mathrm{F}}-L_{\mathrm{MR}}\right)^{2}\right) q r \\
& -2 I_{11}^{\mathrm{MR}} \omega_{\mathrm{MR}} q \\
F_{5}^{*}= & -\left(I_{22}^{\mathrm{F}}+I_{11}^{\mathrm{MR}}-\left(L_{\mathrm{MR}}-L_{\mathrm{F}}\right)^{2} \frac{m_{\mathrm{F}} m_{\mathrm{MR}}}{M}\right) \dot{q} \\
& +2_{11}^{\mathrm{MR}} \omega_{\mathrm{MR}} p \\
& +\left(I_{33}^{\mathrm{F}}+I_{11}^{\mathrm{MR}}-I_{11}^{\mathrm{F}}+\left(L_{\mathrm{F}}-L_{\mathrm{MR}}\right)^{2} \frac{m_{\mathrm{F}} m_{\mathrm{MR}}}{M}\right) p r \\
F_{6}^{*}= & \left(I_{11}^{\mathrm{F}}-I_{22}^{\mathrm{F}}\right) p q-\left(2 I_{11}^{\mathrm{MR}}+I_{33}^{\mathrm{F}}\right) \dot{r}
\end{aligned}
$$

At last, the dynamical equations of translation and rotation can be deduced by Kane's equation $F_{r}+F_{r}^{*}=0$ for $r=1,2, \ldots, 6$.

The dynamical equations of translation can be written as

$$
\begin{aligned}
M \dot{u}= & F_{3}^{\mathrm{MR}}(\sin (\phi) \sin (\psi)+\sin (\theta) \cos (\phi) \cos (\psi)) \\
& -F_{2}^{\mathrm{TR}}(\sin (\psi) \cos (\phi)-\sin (\phi) \sin (\theta) \cos (\psi)) \\
M \dot{v}= & F_{2}^{\mathrm{TR}}(\cos (\phi) \cos (\psi)+\sin (\phi) \sin (\theta) \sin (\psi)) \\
& -F_{3}^{\mathrm{MR}}(\sin (\phi) \cos (\psi)-\sin (\theta) \sin (\psi) \cos (\phi)) \\
M \dot{w}= & F_{2}^{\mathrm{TR}} \sin (\phi) \cos (\theta)+F_{3}^{\mathrm{MR}} \cos (\phi) \cos (\theta)-M g
\end{aligned}
$$

The dynamical equations of rotation can be written as

Thus 


$$
\begin{aligned}
& \dot{p}=a_{12} q+b_{11}^{u} T_{1}^{\mathrm{MR}}+b_{11}^{v} F_{2}^{\mathrm{TR}}+b_{q r} q r \\
& \dot{q}=a_{21} p+b_{22}^{u} T_{2}^{\mathrm{MR}}+b_{22}^{v} T_{2}^{\mathrm{TR}}+b_{p r} p r \\
& \dot{r}=\mathrm{b}_{31}^{u} F_{2}^{\mathrm{TR}}+b_{33}^{v} T_{3}^{\mathrm{MR}}+b_{p q} p q
\end{aligned}
$$

where

$$
\begin{aligned}
& a_{12}=-\frac{2 M I_{11}^{\mathrm{MR}} \omega_{\mathrm{MR}}}{M\left(I_{11}^{\mathrm{F}}+I_{11}^{\mathrm{MR}}\right)+\left(L_{\mathrm{F}}-L_{\mathrm{MR}}\right)^{2} m_{\mathrm{F}} m_{\mathrm{MR}}}, \\
& b_{11}^{u}=\frac{\left(L_{\mathrm{F}} m_{\mathrm{F}}+L_{\mathrm{MR}} m_{\mathrm{MR}}\right) M}{M\left(I_{11}^{\mathrm{F}}+I_{11}^{\mathrm{MR}}\right)+\left(L_{\mathrm{F}}-L_{\mathrm{MR}}\right)^{2} m_{\mathrm{F}} m_{\mathrm{MR}}} \\
& b_{11}^{v}=\frac{L_{\mathrm{MR}} m_{\mathrm{MR}}}{M\left(I_{11}^{\mathrm{F}}+I_{11}^{\mathrm{MR}}\right)+\left(L_{\mathrm{F}}-L_{\mathrm{MR}}\right)^{2} m_{\mathrm{F}} m_{\mathrm{MR}}}, \\
& b_{\mathrm{qr}}=-\frac{M\left(I_{11}^{\mathrm{MR}}-I_{22}^{\mathrm{F}}+I_{33}^{\mathrm{F}}\right)-\left(L_{\mathrm{F}}-L_{\mathrm{MR}}\right)^{2} m_{\mathrm{F}} m_{\mathrm{MR}}}{M\left(I_{11}^{\mathrm{F}}+I_{11}^{\mathrm{MR}}\right)+\left(L_{\mathrm{F}}-L_{\mathrm{MR}}\right)^{2} m_{\mathrm{F}} m_{\mathrm{MR}}} \\
& a_{21}=-\frac{2 M I_{11}^{\mathrm{MR}} \omega_{\mathrm{MR}}}{M\left(I_{22}^{\mathrm{F}}+I_{11}^{\mathrm{MR}}\right)+\left(L_{\mathrm{F}}-L_{\mathrm{MR}}\right)^{2} m_{\mathrm{F}} m_{\mathrm{MR}}}, \\
& b_{22}^{u}=-\frac{M}{M\left(I_{22}^{\mathrm{F}}+I_{11}^{\mathrm{MR}}\right)+\left(L_{\mathrm{F}}-L_{\mathrm{MR}}\right)^{2} m_{\mathrm{F}} m_{\mathrm{MR}}} \\
& b_{22}^{v}=-\frac{M}{M\left(I_{22}^{\mathrm{F}}+I_{11}^{\mathrm{MR}}\right)+\left(L_{\mathrm{F}}-L_{\mathrm{MR}}\right)^{2} m_{\mathrm{F}} m_{\mathrm{MR}}}, \\
& b_{\mathrm{pr}}=-\frac{M\left(I_{33}^{\mathrm{F}}+I_{11}^{\mathrm{MR}}-I_{11}^{\mathrm{F}}\right)+\left(L_{\mathrm{F}}-L_{\mathrm{MR}}\right)^{2} m_{\mathrm{F}} m_{\mathrm{MR}}}{M\left(I_{22}^{\mathrm{F}}+I_{11}^{\mathrm{MR}}\right)+\left(L_{\mathrm{F}}-L_{\mathrm{MR}}\right)^{2} m_{\mathrm{F}} m_{\mathrm{MR}}} \\
& b_{31}^{u}=-\frac{L_{\mathrm{T}}}{I_{33}^{\mathrm{FF}}+2 I_{11}^{\mathrm{MR}}}, \quad b_{33}^{v}=\frac{1}{I_{33}^{\mathrm{F}}+2 I_{11}^{\mathrm{MR}}}, \\
& b_{\mathrm{pq}}=\frac{I_{11}^{\mathrm{F}}-I_{22}^{\mathrm{F}}}{I_{33}^{\mathrm{F}}+2 I_{11}^{\mathrm{MR}}}
\end{aligned}
$$

\section{LINEARIZATION AND ANALYSIS OF THE MODEL}

From the derived kinematic and dynamic equations it is possible to observe that equations (12), (13), and (14) are non-linear equations, unlike equation (11). Also, there is coupling in these equations. However, according to the assumptions, equation (14) is linear as $r$ and $\omega_{\mathrm{MR}}$ are constant. The equations (12a) and (12b) are also linear during hovering or low speed operation. Because the pitch angle and roll angle are very small, which means that $\cos (\phi) \approx 1, \quad \cos (\theta) \approx 1, \quad \sin (\phi) \approx \phi, \quad \sin (\theta) \approx \theta$, equations (12a) and (12b) can be simplified to

$$
\begin{aligned}
& \dot{\phi}=p \\
& \dot{\theta}=q
\end{aligned}
$$

This is a linearized model. It should be noted that the model presented in [4] and [5] can not be linearized easily. The rotational kinematic equations presented in [4] and [5] are

$$
\begin{aligned}
& \dot{q}_{4}=\left(u_{4} \cos \left(q_{6}\right)-u_{5} \sin \left(q_{6}\right)\right) / \cos \left(q_{5}\right) \\
& \dot{q}_{5}=u_{4} \sin \left(q_{6}\right)+u_{5} \cos \left(q_{6}\right) \\
& \dot{q}_{6}=u_{6}+\tan \left(q_{5}\right)\left(u_{4} \cos \left(q_{6}\right)-u_{5} \sin \left(q_{6}\right)\right)
\end{aligned}
$$

where, $q_{4}, q_{5}, q_{6}$ are the Euler angles 1-2-3, and $u_{4}, u_{5}$, $u_{6}$ are the angular speeds in the frame $\mathrm{F}$ of the fuselage.

From the above equation, we can see that $\dot{q}_{4}, \dot{q}_{5}$ are affected by $q_{6}$; however, $q_{6}$ may change during flight. Thus it is not a good idea to linearize the model.

The model of the helicopter considered as one rigid body is also deduced in order to isolate differences between them. The results show that the kinematic equations and dynamic equations of translation are the same. However, the dynamic equations of rotation are different.

The following equations describe the dynamics of rotation considering the helicopter as one rigid body

$$
\begin{aligned}
& \dot{p}=\frac{1}{I_{11}}\left(T_{1}^{\mathrm{MR}}-L_{\mathrm{F}} F_{2}^{\mathrm{TR}}\right)-\frac{I_{33}-I_{22}}{I_{11}} q r \\
& \dot{q}=\frac{1}{I_{22}}\left(T_{2}^{\mathrm{MR}}+T_{2}^{\mathrm{TR}}\right)-\frac{I_{11}-I_{33}}{I_{22}} p r \\
& \dot{r}=\frac{1}{I_{33}} T_{3}^{\mathrm{MR}}-\frac{I_{22}-I_{11}}{I_{33}} p q-\frac{L_{\mathrm{T}}}{I_{33}} F_{2}^{\mathrm{TR}}
\end{aligned}
$$

where, $I_{11}, I_{22}, I_{33}$ are the principal moments of the inertia of the helicopter.

It can be seen that equation (17) is different to equation (14). In order to simplify the analysis of the difference, $F_{2}^{\mathrm{TR}}$ and $T_{2}^{\mathrm{TR}}$ are considered as disturbances and $r$ is taken to be zero. The equations (14a) and (14b) become

$$
\begin{aligned}
& \dot{p}=a_{12} q+b_{11}^{u} T_{1}^{\mathrm{MR}} \\
& \dot{q}=a_{21} p+b_{22}^{u} T_{2}^{\mathrm{MR}}
\end{aligned}
$$

The equations (17a) and (17b) simplify to

$$
\begin{gathered}
\dot{p}=\frac{T_{1}^{\mathrm{MR}}}{I_{11}} \\
\dot{\boldsymbol{q}}=\frac{T_{2}^{\mathrm{MR}}}{I_{22}}
\end{gathered}
$$

Comparing equation (18) with equation (19), it is clear that they are different. In particular, there exist the 


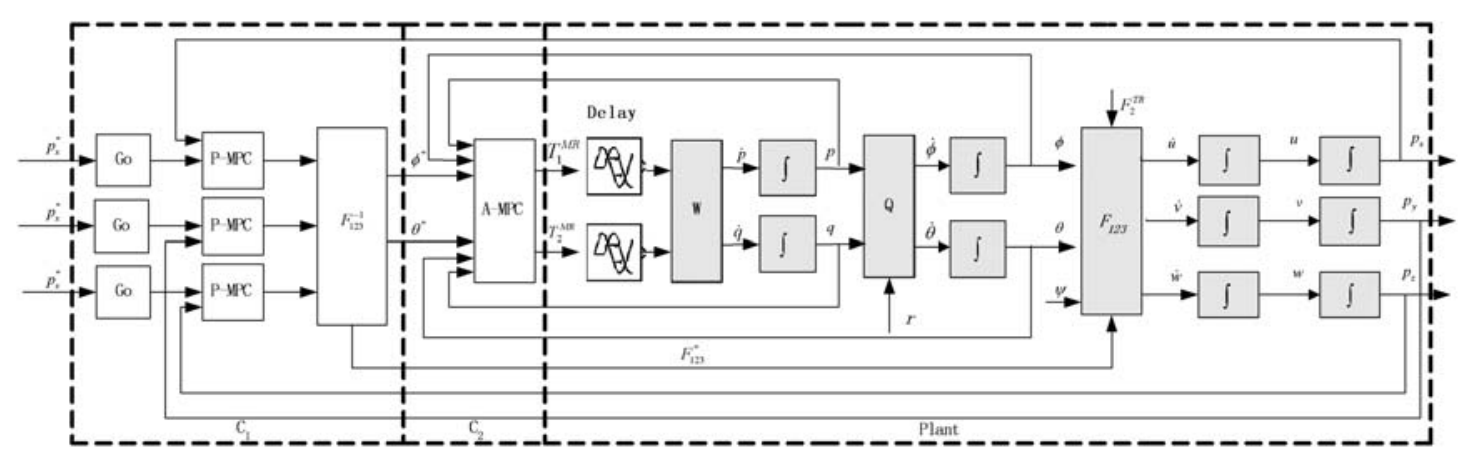

Fig. 2 Scheme of the helicopter controller

terms $a_{12}, a_{21}$ in equation (18), while there are no such terms in equation (19), which make strong coupling between the lateral and longitudinal channels. This becomes clear by solving equations (18) and (19) if the input $T_{1}^{\mathrm{MR}}, T_{2}^{\mathrm{MR}}$ are considered as being constant.

For equation (18)

$$
\begin{aligned}
& p=\frac{b_{22}^{u}}{a_{12}} T_{2}^{\mathrm{MR}}+C_{1} \cos \left(a_{12} t\right)+C_{2} \sin \left(a_{12} t\right) \\
& q=-\frac{b_{11}^{u}}{a_{12}} T_{1}^{\mathrm{MR}}+C_{2} \cos \left(a_{12} t\right)-C_{1} \sin \left(a_{12} t\right)
\end{aligned}
$$

For equation (19)

$$
\begin{aligned}
& p=\frac{1}{I_{11}} T_{1}^{\mathrm{MR}} t+C_{1} \\
& q=\frac{1}{I_{22}} T_{2}^{\mathrm{MR}} t+C_{2}
\end{aligned}
$$

It is clear that the results are completly different. The step response of $p$ and $q$ is cosine according to equation (20), whereas the step response of $p$ and $q$ is linear according to equation (21) under the above assumptions. As a result, we should model the helicopter as two rigid bodies not as one rigid body.

\section{DESIGN OF THE CONTROLLER}

After the linearization described in section 4, equations (14) and (15) are linear and only equation (13) is non-linear. It can be linearized using the dynamic inversion method. After the linearization, the design of the flight controller will become facile. An MPC which has many advantages is applied to control the helicopter.

The general scheme of the helicopter controller is shown in Fig. 2. In the figure, $C_{1}$ denotes the position controller, $C_{2}$ the attitude controller, and Plant the helicopter or its model. The block W denotes the rotational dynamics (14), the block Q denotes the rotational kinematics (12), and the block $\mathrm{F}_{123}$ denotes the translational dynamics (13). The block A-MPC is the attitude MPC controller which controls the angles $\phi, \theta$. The combination of the block $\mathrm{G}_{0}, \mathrm{P}-\mathrm{MPC}$, and the block $\mathrm{F}_{123}^{-1}$ is the position controller which controls the position $p_{x}, p_{\mathrm{y}}, p_{\mathrm{z}}$. The block $G_{0}$, is the pre-filter which softens the trajectory and the block P-MPC generates the desired accelerations. The block $\mathrm{F}_{123}^{-1}$ is the inversion of equation (13). As for the control of the azimuth, it is implemented through a separate control loop using a PID controller based on the angular vector control system gyro equipped on the helicopter.

In order to design the A-MPC, we need to know the state space equations. Considering that $r$ is equal to zero, $F_{2}^{\mathrm{TR}}$ and $T_{2}^{\mathrm{TR}}$ are disturbances, the combination of equations (14a), (14b), and (15) will be

$\left[\begin{array}{c}\dot{\phi} \\ \dot{\theta} \\ \dot{p} \\ \dot{q}\end{array}\right]=\left[\begin{array}{cccc}0 & 0 & 1 & 0 \\ 0 & 0 & 0 & 1 \\ 0 & 0 & 0 & a_{12} \\ 0 & 0 & a_{21} & 0\end{array}\right]\left[\begin{array}{l}\phi \\ \theta \\ p \\ q\end{array}\right]+\left[\begin{array}{cc}0 & 0 \\ 0 & 0 \\ b_{11}^{u} & 0 \\ 0 & b_{22}^{u}\end{array}\right]\left[\begin{array}{l}T_{1}^{\mathrm{MR}} \\ T_{2}^{\mathrm{MR}}\end{array}\right]$

$y=\left[\begin{array}{llll}1 & 0 & 0 & 0 \\ 0 & 1 & 0 & 0 \\ 0 & 0 & 1 & 0 \\ 0 & 0 & 0 & 1\end{array}\right]\left[\begin{array}{l}\phi \\ \theta \\ p \\ q\end{array}\right]$

This is the state space equation of the attitude part of the model. The time delay of the plant is $0.12 \mathrm{~s}$ and the limits of actuators are $-6 \mathrm{Nm} \leqslant u \leqslant 6 \mathrm{Nm}$. The error weight matrix $\mathbf{S}$ is $\operatorname{diag}(10,10,0,0)$ and the control weight matrix $\mathbf{T}$ equals $\operatorname{diag}(0.01,0.01)$. The predictive horizon $N=30$ and control horizon $N_{u}=2$. Substituting the parameters of the helicopter into equation (22), we get $a_{12}=10.17, a_{21}=-10.17$, $b_{11}^{u}=1.95, b_{22}^{u}=1.95$.

Thus, all of the coefficients of equation (2) are known, and it can be transformed into the form of equation (3). The matrices $\mathbf{Q}, \mathbf{C}, \mathbf{G}, \mathbf{W}, \mathbf{S}$ have the following forms 


$$
\begin{aligned}
& \mathbf{Q}=\left[\begin{array}{cccc}
0.644 & 0 & 0.409 & -0.014 \\
0 & 0.644 & 0.014 & 0.409 \\
0.409 & 0.0134 & 0.529 & 0 \\
-0.014 & 0.409 & 0 & 0.529
\end{array}\right] \quad \mathbf{C}=\left[\begin{array}{cccc}
25.568 & -11.99 & \cdots & 0 \\
11.99 & 25.258 & \cdots & 0 \\
21.811 & -9.585 & \cdots & 0 \\
9.585 & 21.811 & \cdots & 0
\end{array}\right]_{4 \times 34} \\
& \mathbf{G}=\left[\begin{array}{cccc}
1 & 0 & 0 & 0 \\
0 & 1 & 0 & 0 \\
-1 & 0 & 0 & 0 \\
0 & -1 & 0 & 0 \\
1 & 0 & 1 & 0 \\
0 & 1 & 0 & 1 \\
-1 & 0 & -1 & 0 \\
0 & -1 & 0 & -1
\end{array}\right], \quad \mathbf{W}=\left[\begin{array}{l}
6 \\
6 \\
6 \\
6 \\
6 \\
6 \\
6 \\
6
\end{array}\right], \quad \mathbf{S}=\left[\begin{array}{cccccc}
0 & \cdots & -1 & 0 & \cdots & 0 \\
0 & \cdots & 0 & -1 & \cdots & 0 \\
0 & \cdots & 1 & 0 & \cdots & 0 \\
0 & \cdots & 0 & 1 & \cdots & 0 \\
0 & \cdots & -1 & 0 & \cdots & 0 \\
0 & \cdots & 0 & -1 & \cdots & 0 \\
0 & \cdots & 1 & 0 & \cdots & 0 \\
0 & \cdots & 0 & 1 & \cdots & 0
\end{array}\right]_{8 \times 34}
\end{aligned}
$$

This QP problem can be solved by the explicit MPC described in section 2. Through the explicit MPC algorithm, the QP problem will be changed to look-up the corresponding feedback gain. The following equation is the solution to the QP problem. There are 41 polyhedral portions $R_{1}, R_{2} \ldots R_{41}$

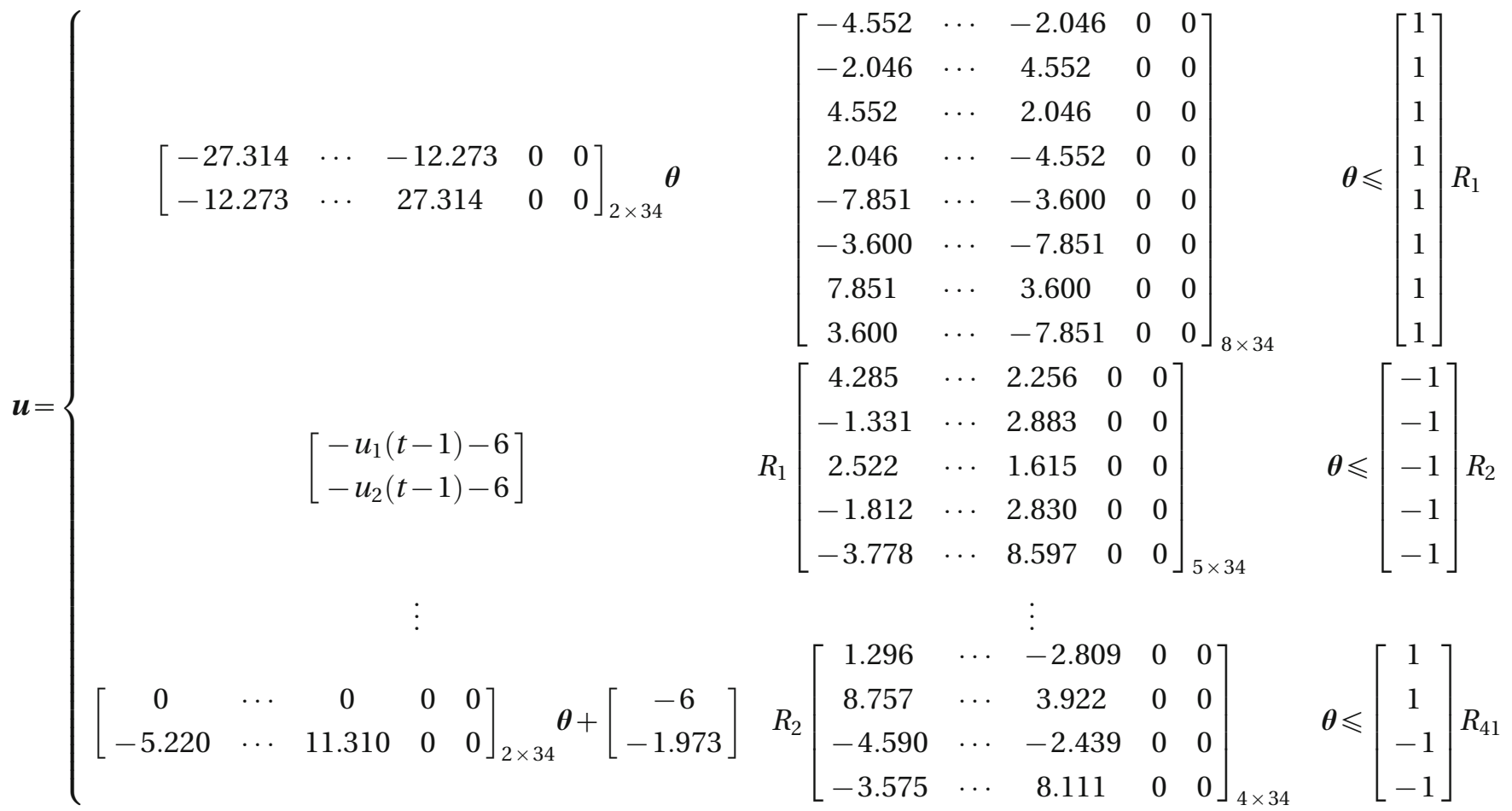




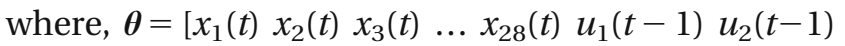
$\left.r_{1}(t) r_{2}(t) r_{3}(t) r_{4}(t)\right]^{\prime}$

It should be noted that there are 24 more state variables than usual because of the time delay $(0.12 \mathrm{~s})$.

As for the position control, it is very hard to design a model predictive controller because of the non-linearity of the translational dynamics. However, the translational dynamics can be linearized by its inversion, $\mathrm{F}_{123}^{-1}$. Unfortunately these equations are transcendental equations and hard to solve. However, when the helicopter hovers or flies at low speeds, the pitch and roll angles are very small. Then equation (13) can be rewritten as

$$
\begin{aligned}
& M \dot{u}=F_{3}^{\mathrm{MR}}(\phi \sin (\psi)+\theta \cos (\psi))-F_{2}^{\mathrm{TR}} \sin (\psi) \\
& M \dot{v}=F_{2}^{\mathrm{TR}} \cos (\psi)-F_{3}^{\mathrm{MR}}(\phi \cos (\psi)-\theta \sin (\psi)) \\
& M \dot{\boldsymbol{w}}=F_{2}^{\mathrm{TR}} \phi+F_{3}^{\mathrm{MR}}-M g
\end{aligned}
$$

with its inversion being

$$
\begin{aligned}
\phi & =\frac{M(\dot{u} \sin \psi-\dot{v} \cos \psi)+F_{2}^{\mathrm{TR}}}{F_{3}^{\mathrm{MR}}} \\
\theta & =\frac{M(\dot{u} \cos (\psi)+\dot{v} \sin (\psi))}{F_{3}^{\mathrm{MR}}}
\end{aligned}
$$

where

$$
F_{3}^{\mathrm{MR}}=\sqrt{\left.M^{2}\left(\dot{u}^{2}+\dot{v}^{2}+(\dot{w}+g)^{2}\right)-\left(F_{2}^{\mathrm{TR}}\right)^{2}\right)}
$$

Thus, the combination of the block $\mathrm{F}_{123}^{-1}$, the attitude controller, and the helicopter will be three double integrators. The position of the helicopter can be controlled by three independent explicit model predictive controllers, P-MPCs.

The state space equation for the block P-MPC is

$$
\begin{aligned}
& \dot{\boldsymbol{x}}=\left[\begin{array}{ll}
0 & 1 \\
0 & 0
\end{array}\right] \boldsymbol{x}+\left[\begin{array}{l}
0 \\
1
\end{array}\right] \boldsymbol{u} \\
& \boldsymbol{y}=\left[\begin{array}{ll}
1 & 0
\end{array}\right] \boldsymbol{x}
\end{aligned}
$$

The limits are

$$
-10 \mathrm{Nm} \leqslant u \leqslant 10 \mathrm{Nm}
$$

The error weight matrix $\mathbf{S}=10$, the control weight error matrix $\mathbf{T}=0.1$, the predictive horizon $N=40$, the control horizon $N u=3$.

The matrices $\mathbf{Q}, \mathbf{C}, \mathbf{G}, \mathbf{W}, \mathbf{S}$ are

$$
\begin{aligned}
& \mathbf{Q}=\left[\begin{array}{lll}
0.600 & 0.241 & 0.218 \\
0.241 & 0.555 & 0.199 \\
0.218 & 0.199 & 0.517
\end{array}\right] \\
& \mathbf{C}=\left[\begin{array}{cccc}
4.936 & -4.787 & \cdots & -11.644 \\
4.450 & -4.317 & \cdots & -10.419 \\
3.995 & -3.876 & \cdots & -9.283
\end{array}\right]_{3 \times 16} \\
& \mathbf{G}=\left[\begin{array}{ccc}
1 & 0 & 0 \\
-1 & 0 & 0 \\
1 & 1 & 0 \\
-1 & -1 & 0 \\
1 & 1 & 1 \\
-1 & -1 & -1
\end{array}\right] \\
& \mathbf{W}=\left[\begin{array}{c}
10 \\
10 \\
10 \\
10 \\
10 \\
10
\end{array}\right]
\end{aligned}
$$

$$
\mathbf{S}=\left[\begin{array}{ccccc}
0 & \cdots & 0 & -1 & 0 \\
0 & \cdots & 0 & 1 & 0 \\
0 & \cdots & 0 & -1 & 0 \\
0 & \cdots & 0 & 1 & 0 \\
0 & \cdots & 0 & -1 & 0 \\
0 & \cdots & 0 & 1 & 0
\end{array}\right]_{6 \times 16}
$$

This QP problem can be solved as the A-MPC. The following equation is the solution to the QP problem, which has 13 polyhedral portions $R_{1}, R_{2} \ldots R_{13}$ 


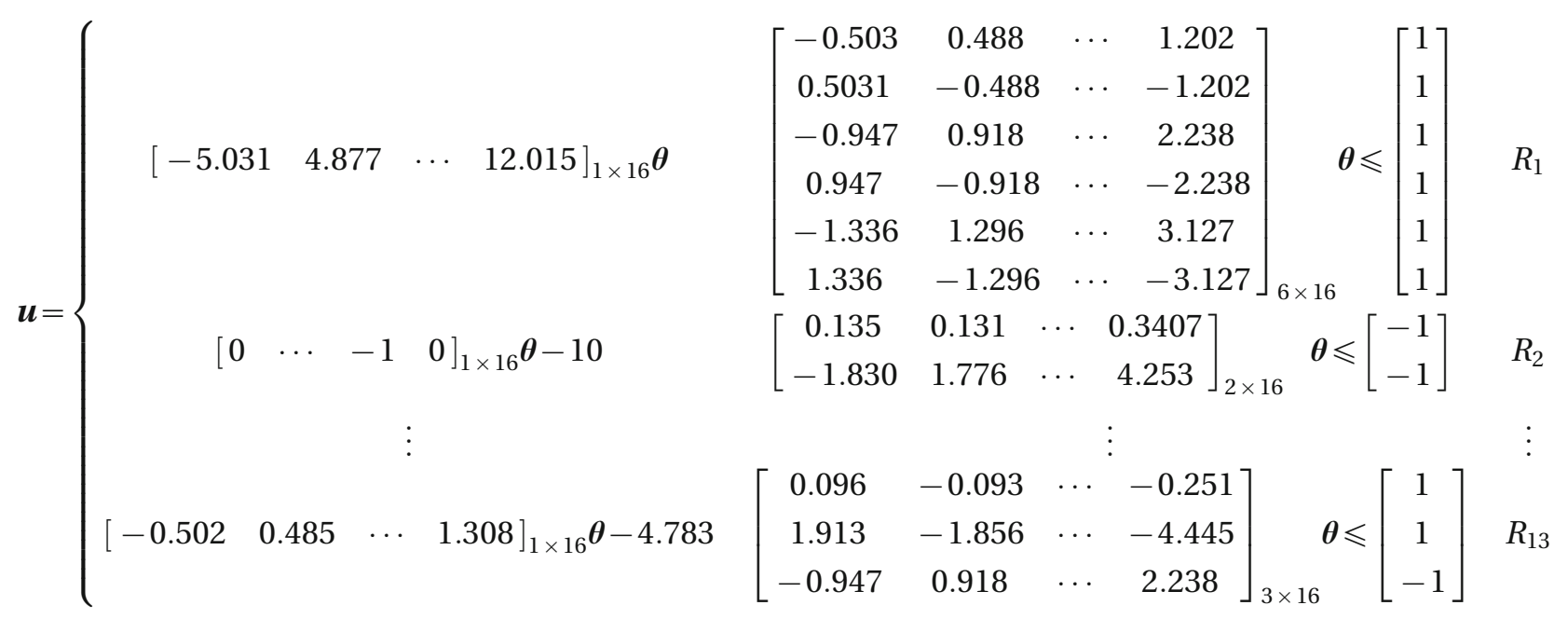

\section{REAL FLIGHT EXPERIMENTS}

In order to validate the controller, experiments were performed in the laboratory for autonomously flying robots. Figure 3 shows the flight scene. The helicopter is fixed in a safety cage which is made of carbon tubes and has a mass of $1.2 \mathrm{~kg}$. The four high brightness lamps (markers) are used to measure the actual position and orientation of the helicopter using a vision system.

The control algorithm runs on a control computer with an $800 \mathrm{MHz}$ CPU, a 128M RAM, two CAN-bus interfaces, three RS-232 interfaces, one RS-485 interface, and an Ethernet interface. The onboard microcontroller which was based on a Siemens SAB80C167 microcontroller deals with the angle velocity signals measured by the ADXRS 300 gyroscopes and transferred to the control computer over the CAN-bus. The three-camera vision system (the three cameras are placed on the ceiling of the laboratory) measures the position and orientation of the helicopter which is sent to the ground computer through the Ethernet.

In the flight experiment, the helicopter started at position $(0,-1.5,0.9)$, then moved to the position ( 1 ,

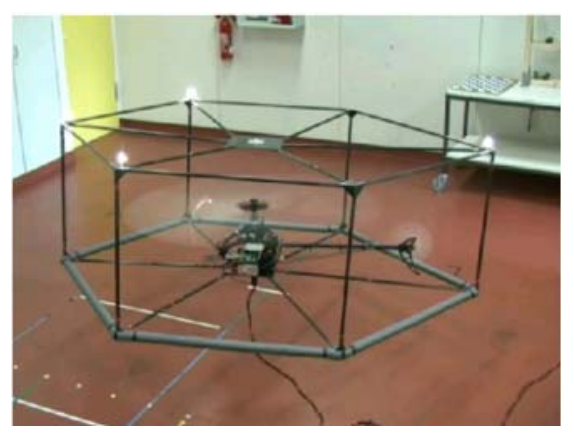

Fig. 3 The flight scene
$-1.5,0.9)$, then to the original position. Figures 4 to 6 show the controller performance. From the figures, we can see that the step response has no overshoot and the position error is $\pm 0.15 \mathrm{~m}$ on the $x$ axis, and $\pm 0.15 \mathrm{~m}$ on the $y$ axis, $\pm 0.05 \mathrm{~m}$ on the $z$ axis. The maximum error is $\pm 0.15 \mathrm{~m}$ which is accurate enough for most practical applications.

\section{CONCLUSIONS}

The constructed mathematical model regards the helicopter as consisting of two rigid bodies, the fuselage and the main rotor. The model is constructed considering the inertial effects of the main rotor. MPC can deal with multivariables, coupling, constraints, and time delay and can be utilized in the control of helicopters. The explicit MPC does not need significant computation and can run on the onboard computer and is applied to control a toy helicopter. The real flight experimental results show that the controller achieves a good performance and can meet the requirements for application. The helicopter can now fly well in laborary condititions and outdoor

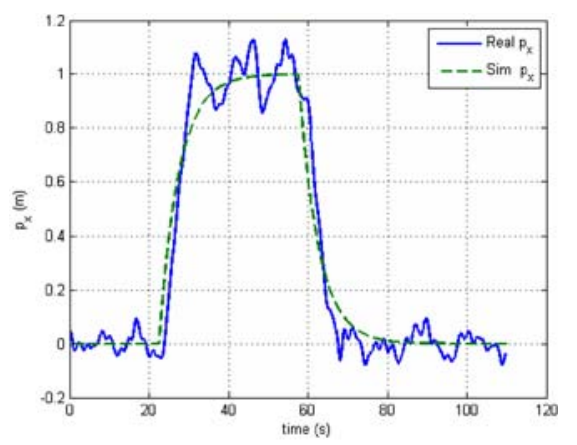

Fig. 4 Coordinate $p_{x}$ of the helicopter 


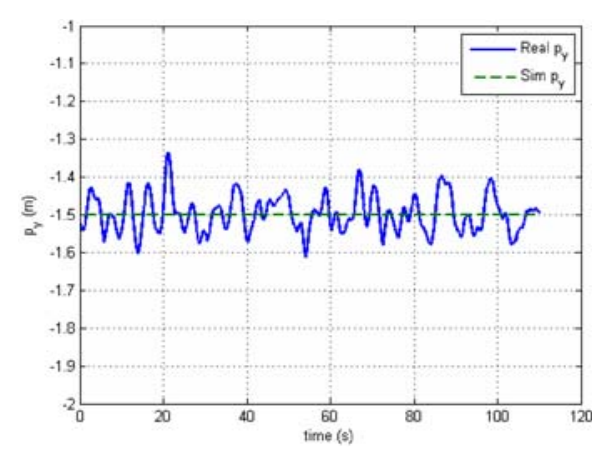

Fig. 5 Coordinate $p_{y}$ of the helicopter

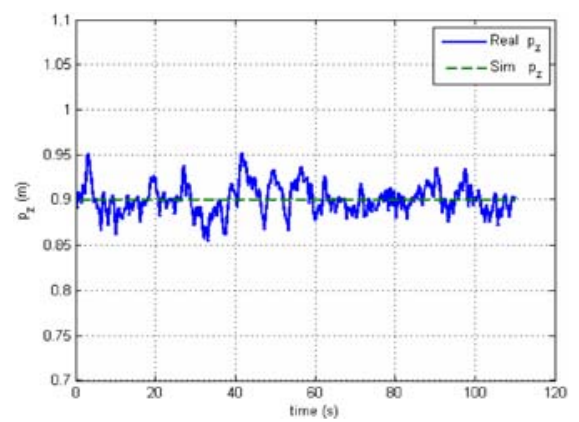

Fig. 6 Coordinate $p_{z}$ of the helicopter

experiments to validate its performance in a more complex environment are planned in the future.

\section{ACKNOWLEDGEMENTS}

This research is a Project-Based Personnel Exchange Programme and supported by the German Academic Exchange Service and the China Scholarship Council. The authors would like to thank Markus Bernard and Maximilian Laiacker for the help in the flight experiments and the anonymous reviewers for improving the quality of the paper.

\section{REFERENCES}

1 Zhou, J.-J., Chen, C. and Cui, M.-H. Development and military application of unmanned helicopters. Aeronaut. Sci. Technol., 2003, (1), 38-40.

2 Zhang, Y.-O., Lü, T.-S. and Du, J.-F. The pitch and roll attitude modeling and stable control of unmanned helicopter. J. Shanghai Jiaotong Uni., 2007, 41(1), 100-103.

3 Xu, M.-Y. and Jin, S. B. Flight dynamics, 2004 (Science Press, Beijing).

4 Gao, Z. and Chen, R. L. Helicopter flight dynamics, 2003 (Science Press, Beijing).

5 Sung, K. K. Modeling, identification, and trajectory planning for a model-scale helicopter. $\mathrm{PhD}$ Thesis, University of Michigan, Ann Arbor, MI, 2001.
6 Mettler, B. Modeling small-scale unmanned rotorcraft for advanced flight control design. $\mathrm{PhD}$ Thesis, Carnegie Mellon University, Pittsburgh, PA, 2001.

7 Sun, T. and Song, Y. Study on a method for unmanned helicopter flight dynamics model identification. Acta Aeronaut. Astronaut. Sinica, 2007, 28(1), S14-S18.

8 Wu, J.-D., Li, P. and Han, B. A modeling method of miniature unmanned helicopter based on parameter identification. Acta Aeronaut. Astronaut. Sinica, 2007, 28(4), 845-850.

9 Kondak, K., Deeg, C., Hommel, G., Musial, M. and Remuß, V. Mechanical model and control of an autonomous small size helicopter with a stiff main rotor. In Proceedings of the IEEE/RSJ International Conference on Intelligent robots and systems, Volume 3, 2004, pp. 2469-2474.

10 Kondak, K., Bernard, M., Losse, N. and Hommel, G. Elaborated modeling and control for autonomous small size helicopters. VDI Berichte, 2006, 1956, 207-219.

11 Tait-Bryan angles, http://en.wikipedia.org/wiki/ Tait-Bryan_angles, 2007.

12 Sanders, C. P. and DeBitettoc, P. A. Hierarchical control of small autonomous helicopters. In Proceedings of the 37th IEEE Conference on Decision and control, Tampa, Florida, USA, 1998, pp. 3629-3633.

13 Sasaki, M., Ishida, H., Katsuno, T. and Ogasawara, A. Learning fuzzy logic controller for hovering a helicopter. In Proceedings of the 1998 Conference of the North American Fuzzy Processing Society - NAFIPS, Pensacola Beach, FL, USA, pp. 25-28.

14 Nakanishi, H. and Inoue, K. Development of autonomous flight control system for unmanned helicopter by use of neural networks. In Proceedings of the International Joint Conference on Neural networks, Portland, OR, USA, Vol. 3, 2003, pp. 2400-2405 (IEEE).

15 Camacho, E. F. and Bordons, C. Model predictive control, 1999 (Springer-Verlag).

16 Dutka, A. S., Ordys, A. W. and Grimble, M. J. Nonlinear predictive control of 2DOF helicopter model. In Proceedings of the 42nd IEEE Conference on Decision and control, Maui, HI, 2003, pp. 3954-3958.

17 Balderud, J. and Wilon, D. I. Application of predictive control to a toy helicopter. In Proceedings of the 2002 IEEE International Conference on Control applications, Glasgow, Scotland, 2002, pp. 1225-1229.

18 Bemporad, A., Morari, M., Dua, V. and Pistikopoulos, E. N. The explicit linear quadratic regulator for constrained systems. Automatica, 2002, 38, 3-20.

\section{APPENDIX}

\section{Notation}

$\boldsymbol{f}_{1}, \boldsymbol{f}_{2}, \boldsymbol{f}_{3}$
$F_{2}^{\mathrm{TR}}$

unit vectors

force generated by the tail rotor 


\begin{tabular}{|c|c|c|c|}
\hline$F_{3}^{\mathrm{MR}}$ & $\begin{array}{l}\text { lifting force generated by the main } \\
\text { rotor } \\
\text { generalized active force }\end{array}$ & $p, q, r$ & $\begin{array}{l}\text { rotation speeds with respect to body } \\
\text { frame around the } f_{1}, f_{2} \text {, and } f_{3} \text { axes } \\
\text { respectively }\end{array}$ \\
\hline & generalized inertial force & $p_{\mathrm{x}}, p_{\mathrm{y}}, p_{\mathrm{z}}$ & coordinates of the helicopter in the \\
\hline$I_{11}^{\mathrm{F}}, I_{22}^{\mathrm{F}}, I_{33}^{\mathrm{F}}$ & $\begin{array}{l}\text { principal moments of the inertia of } \\
\text { the fuselage around the } f_{1}, f_{2}, f_{3} \text { axes }\end{array}$ & & $\begin{array}{l}\text { inertial frame in } \mathrm{f}_{1}, \mathrm{f}_{2} \text {, and } \mathrm{f}_{3} \text { direc- } \\
\text { tions respectively }\end{array}$ \\
\hline \multirow[t]{2}{*}{$I_{11}^{\mathrm{MR}}, I_{22}^{\mathrm{MR}}, I_{33}^{\mathrm{MR}}$} & $\begin{array}{l}\text { respectively } \\
\text { principal moments of inertia of main }\end{array}$ & $T_{1}^{\mathrm{MR}}$ & $\begin{array}{l}\text { torques generated by main rotor in } f_{1} \\
\text { axis }\end{array}$ \\
\hline & $\begin{array}{l}\text { rotor around the } f_{1}, f_{2}, f_{3} \text { axes respec- } \\
\text { tively }\end{array}$ & $T_{2}^{\mathrm{MR}}$ & $\begin{array}{l}\text { torques generated by main rotor in } f_{2} \\
\text { axis }\end{array}$ \\
\hline$L_{\mathrm{F}}$ & $\begin{array}{l}\text { offset of the point } \mathrm{O}_{\mathrm{F}} \text { from the point } \\
\text { Fo in the } f_{3} \text { direction in Fig. } 1\end{array}$ & $T_{3}^{\mathrm{MR}}$ & $\begin{array}{l}\text { torques generated by main rotor in } f_{3} \\
\text { axis }\end{array}$ \\
\hline$L_{\mathrm{MR}}$ & $\begin{array}{l}\text { offset of the point } \mathrm{O}_{\mathrm{F}} \text { from the point } \\
\text { MRo in the } f_{3} \text { direction in Fig. } 1\end{array}$ & $T_{2}^{\mathrm{TR}}$ & drag torques generated by tail rotor \\
\hline$L_{\mathrm{T}}$ & $\begin{array}{l}\text { offset of the point } \mathrm{O}_{\mathrm{F}} \text { from the point } \\
\text { TRo in } f_{1} \text { direction in Fig. } 1\end{array}$ & $u, v, l$ & $\begin{array}{l}\text { in the inertial frame in } f_{1}, f_{2} \text {, and } f_{3} \\
\text { directions respectively }\end{array}$ \\
\hline $\begin{array}{l}m_{\mathrm{F}} \\
m_{\mathrm{MR}}\end{array}$ & $\begin{array}{l}\text { mass centre of the fuselage } \\
\text { mass centre of the main rotor }\end{array}$ & $\phi, \theta, \psi$ & roll angle, pitch angle, and yaw angle \\
\hline & mass centre of helicopter & & \\
\hline & unit vectors & $\omega_{\mathrm{MR}}$ & rotation speed of main rotor \\
\hline
\end{tabular}

